\title{
A NATURAL REPRESENTATION OF PARTITIONS AS TERMS OF A UNIVERSAL ALGEBRA
}

\author{
HARRY LAKSER
}

(Received September 17 1990)

Communicated by T. E. Hall

\begin{abstract}
We consider a variety of algebras with two binary commutative and associative operations. For each integer $n \geq 0$, we represent the partitions on an $n$-element set as $n$-ary terms in the variety. We determine necessary and sufficient conditions on the variety ensuring that, for each $n$, these representing terms be all the essentially $n$-ary terms and moreover that distinct partitions yield distinct terms.

1991 Mathematics subject classification (Amer. Math. Soc.): primary: 08 A 40; secondary 08 B 20,05 A 17.

Keywords and phrases: Bell bisemigroup, essentialy $n$-ary, partition, variety.
\end{abstract}

\section{Introduction}

Following the notation of [4], we denote the set of $n$-ary term operations on an algebra $\mathbf{A}$ by $\mathrm{Clo}_{n} \mathbf{A}$. We say that an $n$-ary term $f\left(x_{0}, \ldots, x_{n-1}\right)$ does not depend on the variable $x_{i}$ in $\mathbf{A}$ if the identity

$$
f\left(x_{0}, \ldots, x_{i-1}, y, x_{i+1}, \ldots, x_{n-1}\right)=f\left(x_{0}, \ldots, x_{i-1}, z, x_{i+1}, \ldots, x_{n-1}\right)
$$

is satisfied in the algebra $\mathbf{A}$. Otherwise, we say that $f$ depends on $x_{i}$. Similarly, if $\mathscr{V}$ is a variety of algebras, we say that the term $f\left(x_{0}, \ldots, x_{n-1}\right)$ does not

(C) 1993 Australian Mathematical Society 0263-6115/93 \$A2.00+0.00

This research was supported by the NSERC of Canada 
depend on the variable $x_{i}$ in $\mathscr{V}$ if $\mathscr{V}$ satisfies the identity

$$
f\left(x_{0}, \ldots, x_{i-1}, y, x_{i+1}, \ldots, x_{n-1}\right)=f\left(x_{0}, \ldots, x_{i-1}, z, x_{i+1}, \ldots, x_{n-1}\right)
$$

We say that the $n$-ary term $f\left(x_{0}, \ldots, x_{n-1}\right)$ is essentially $n$-ary if $f$ depends on all the variables $x_{0}, \ldots, x_{n-1}$. Following G. Grätzer [2], we denote by $P_{n}(\mathrm{~A})$ the subset of $\mathrm{Clo}_{n} \mathbf{A}$ consisting of all essentially $n$-ary term operations of $\mathbf{A}$.

We denote the set of equivalence relations on a set $X$ by Eqv $X$ and, without further ado, think of them interchangeably as either equivalence relations or as partitions of $X$. Note that Eqv $\emptyset=\{\emptyset\}$. We denote by $\mathbb{N}_{n}$ the set $\{0, \ldots, n-1\}$. Note that $\mathbb{N}_{0}=\emptyset$. We denote by $\mathbb{N}$ the set $\{0,1, \ldots\}$ of all natural numbers.

By a commutative bisemigroup $\mathbf{A}$ we mean an algebra $\langle A,+, \cdot\rangle$ such that + and - are binary commutative and associative operations. Let $\mathbf{A}$ be a commutative bisemigroup with a nullary term denoted 0 - whether 0 is a nullary fundamental operation or the value of a constant term of arity $>0$ will turn out to be irrelevant. For each integer $n \geq 0$ we define a mapping

$$
\Phi_{n}: \mathrm{Eqv} \mathbb{N}_{n} \rightarrow \mathrm{Clo}_{n} \mathbf{A}
$$

by setting

$$
\Phi_{0}(\emptyset)=0
$$

and, for $n>0$ and each $\alpha \in$ Eqv $\mathbb{N}_{n}$, by setting

$$
\Phi_{n}(B)=\prod\left(x_{i} \mid i \in B\right)
$$

for each block $B$ of the partition $\alpha$, and setting

$$
\Phi_{n}(\alpha)=\sum \Phi_{n}(B),
$$

the sum being taken over all the blocks $B$ of $\alpha$. For example, for the unique partition $\alpha$ of $\mathbb{N}_{1}$, we have

$$
\Phi_{1}(\alpha)=x_{0},
$$

and, for the partition $\alpha=\{\{0,2,4\},\{1\},\{3\}\}$ of $\mathbb{N}_{5}$, we have

$$
\Phi_{5}(\alpha)=x_{0} x_{2} x_{4}+x_{1}+x_{3} .
$$

We say that a commutative bisemigroup $\mathbf{A}$ is a Bell bisemigroup if, for each $n \geq 0$, the representation $\Phi_{n}$ is a bijection between Eqv $\mathbb{N}_{n}$ and $P_{n}(\mathbf{A})$, that is, if the essentially $n$-ary term operations are precisely those term operations 
representing partitions of $\mathbb{N}_{n}$ and distinct partitions yield distinct term operations. We choose this terminology because the cardinality of Eqv $\mathbb{N}_{n}$ is often called the Bell number $B(n)$-see [5, page 33]. Clearly, whether or not the commutative bisemigroup $A$ is a Bell bisemigroup depends only on the variety $\mathscr{V}$ generated by $\mathbf{A}$; consequently, we say that a variety of bisemigroups is a Bell variety of bisemigroups if it is generated by a Bell bisemigroup.

\section{Bell varieties of bisemigroups}

In this section and the next we characterize Bell varieties of bisemigroups. We consider the following identities, where 0 denotes a nullary,

$$
\begin{aligned}
x+y & =y+x \\
x y & =y x \\
(x+y)+z & =x+(y+z) \\
(x y) z & =x(y z) \\
0+x & =0 \\
0 x & =0 \\
x+x & =0 \\
x x & =0 \\
x+x y & =0 \\
x y+x z & =0,
\end{aligned}
$$

and the identities

$$
\begin{aligned}
& (x+y) z=0, \\
& (x+y) z=x y z .
\end{aligned}
$$

In this section we prove:

LEMMA 1. If $\mathscr{V}$ is a Bell variety of bisemigroups, then $\mathscr{V}$ satisfies the identities (1)-(10) and either the identity (11) or (11').

To prove Lemma 1 , let $\mathscr{V}$ be a Bell variety of bisemigroups. Then there is exactly one nullary term,

0 , 
exactly one unary term $f(x)$,

$$
x \text {, }
$$

exactly two essentially binary terms $f(x, y)$,

$$
x+y \text { and } x y \text {, }
$$

and exactly five essentially ternary terms $f(x, y, z)$,

$$
x+y+z, \quad x y z, \quad x+y z, \quad y+x z, \quad x y+z .
$$

Identities (1)-(4) hold by the definition of Bell variety. The key to the proof is the following lemma:

LEMMA 2. $\mathscr{V}$ satisfies one of the identities (11) or (11').

PROOF. If the term $f(x, y, z)=(x+y) z$ does not depend on the variable $z$, then

$$
(x+y) z=(x+y)(u+v)=(u+v)(x+y)=(u+v) w,
$$

that is, $(x+y) z$ is constant, and so we have (11).

If $(x+y) z$ does not depend on $x$, then, by the commutativity of + , it also does not depend on $y$. Consequently, if $(x+y) z$ is not constant, the identity $(x+y) z=z$ holds. But then we have the sequence of identities

$$
x+y=(x+z)(x+y)=(x+y)(x+z)=x+z,
$$

contradicting the fact that $x+y$ is essentially binary.

Consequently, either (11) holds and we are done, or $(x+y) z$ is essentially ternary. In this latter case, by the symmetry in $x$ and $y$ (and the distinctness of the five essentially ternary terms), $\mathscr{V}$ must satisfy one of the identities

$$
\begin{aligned}
& (x+y) z=x y z \\
& (x+y) z=x+y+z \\
& (x+y) z=x y+z .
\end{aligned}
$$

Identity (12) yields

$$
x+y+u v=(x+y) u v=((x+y) u) v=(x+y+u) v=x+y+u+v,
$$

that is, the contradiction

$$
\Phi_{4}(\{\{0\},\{1\},\{2\},\{3\}\})=\Phi_{4}(\{\{0\},\{1\},\{2,3\}\}) .
$$


Similarly, identity (13) yields the contradiction

$$
x y+u v=(x+y) u v=((x+y) u) v=(x y+u) v=x y u+v .
$$

Thus, if $(x+y) z$ is essentially ternary, then identity $\left(11^{\prime}\right)$ holds, concluding the proof of Lemma 2.

We now establish identities (5)-(10). We first consider the unary term $f(x)=$ $x x$. Either (8) holds or we have the identity

$$
x x=x .
$$

But then we have the identity

$$
x+y=(x+y)(x+y) .
$$

If (11) holds, then we get the contradiction

$$
x+y=0 .
$$

If $\left(11^{\prime}\right)$ holds, then we get the contradiction

$$
x+y=x y(x+y)=(x+y) x y=x y x y=x x y y=x y .
$$

Thus, identity (8) is established.

Similarly, if (7) does not hold, then we have the identity

$$
x+x=x .
$$

But then

$$
x y=(x+x) y .
$$

If (11) holds, we get the contradiction

$$
x y=0 .
$$

Similarly, if (11') holds, then, since (8) was established above,

$$
x y=x x y=0 y,
$$

yielding the contradiction that the term $x y$ does not depend on $x$. Thus (7) is established. 
We now establish (6). If (11) holds, then (6) follows immediately from (7):

$$
0 x=(x+x) x=0 .
$$

On the other hand, if $\left(11^{\prime}\right)$ holds and (6) does not hold, then we must have the identity

$$
0 x=x .
$$

But then, using $\left(11^{\prime}\right)$, we get the contradiction

$$
x+y=0(x+y)=(x+y) 0=x y 0=x(y 0)=x(0 y)=x y .
$$

Thus we have established (6).

We now establish (5). If (5) does not hold, then we have the identity

$$
0+x=x .
$$

Then

$$
x y=(0+x) y .
$$

If (11) holds, we get the immediate contradiction

$$
x y=0 .
$$

If $\left(11^{\prime}\right)$ holds, then, by (6), we again get the contradiction

$$
x y=x 0 y=0 .
$$

Thus (5) holds.

We now establish (10). If the term $x y+x z$ does not depend on at least one of the variables $x, y, z$, then, substituting 0 for that variable and using (5) and (6), we get (10). Thus we need only show that the term $x y+x z$ is not essentially ternary. Assume, to the contradictory, that it is. Then, by the symmetry in $y$ and $z, \mathscr{V}$ must satisfy one of the identities

$$
\begin{aligned}
& x y+x z=x+y+z, \\
& x y+x z=x y z, \\
& x y+x z=x+y z .
\end{aligned}
$$

If (14) holds, we get the identity

$$
x+y+z+u=x(y+z)+x u .
$$


If $\mathscr{V}$ satisfies (11), we then get the contradiction

$$
x+y+z+u=0+x u=0 .
$$

If $\mathscr{V}$ satisfies $\left(11^{\prime}\right)$, we get the contradiction

$$
x+y+z+u=x y z+x u=x+y z+u,
$$

using (14) once more to get the second equality. Thus (14) does not hold.

If (15) holds, then we get the contradiction

$$
x y u v=(x y) u+(x y) v=x(y u)+x(y v)=x y u y v=0 x u v=0 .
$$

If (16) holds, then we get the contradiction

$$
\begin{aligned}
x y+u v=(x y) u+(x y) v & =x(y u)+x(y v) \\
& =x+y u y v=x+0 u v=x+0=0 .
\end{aligned}
$$

Consequently, $x y+x z$ is not essentially ternary, and so must be constant, that is, (10) holds.

Finally, we establish (9). If $x+x y$ is not constant, then $\mathscr{V}$ must satisfy one of the identities

$$
\begin{aligned}
& x+x y=x y, \\
& x+x y=x+y, \\
& x+x y=x, \\
& x+x y=y .
\end{aligned}
$$

But then, substituting $u v$ for $x$ and using (10), we get the respective contradictions

$$
\begin{aligned}
& 0=u v y, \\
& 0=u v+y, \\
& 0=u v, \\
& 0=y .
\end{aligned}
$$

Thus (9) holds, concluding the proof of Lemma 1. 


\section{The free Bell bisemigroups}

In this section we prove our main result:

THEOREM. There are precisely two Bell varieties of bisemigroups, the variety $\mathscr{B}$ given by the identities (1)-(11), and the variety $\mathscr{B}^{\prime}$ given by the identities (1)-(10), (11').

In the process of the proof we shall give a natural representation of the free algebras on $\aleph_{0}$ generators in these varieties.

We first note the following two lemmas:

LEMMA 3. Let $\mathscr{V}$ be one of the varieties $\mathscr{B}, \mathscr{B}^{\prime}$, and let $f\left(x_{0}, \ldots, x_{n-1}\right)$ be a term in which the variable $x_{i}, 0 \leq i<n$, appears. Then $\mathscr{V}$ satisfies the identity

$$
f\left(x_{0}, \ldots, x_{i-1}, 0, x_{i+1}, \ldots, x_{n-1}\right)=0 \text {. }
$$

PROOF. The proof follows in a straight-forward manner from identities (5) and (6) by induction on the complexity of the term $f$.

LEMMA 4. Let $\mathscr{V}$ be one of the varieties $\mathscr{B}, \mathscr{B}^{\prime}$, and let $f\left(x_{0}, \ldots, x_{n-1}\right)$ be a term in which all of the variables $x_{0}, \ldots, x_{n-1}$ appear. Then either $\mathscr{V}$ satisfies the identity

$$
f\left(x_{0}, \ldots, x_{n-1}\right)=0
$$

or there is an $\alpha \in E q v \mathbb{N}_{n}$ such that $\mathscr{V}$ satisfies the identity

$$
f\left(x_{0}, \ldots, x_{n-1}\right)=\Phi_{n}(\alpha) .
$$

PROOF. The proof follows in a straight-forward manner from each of the sets of identities (1)-(11) and (1)-(10), (11') by induction on the complexity of the term $f$. More precisely, we prove the following inductively:

Let $n \geq 1$ and let $\varphi: \mathbb{N}_{n} \rightarrow \mathbb{N}$, the set of natural numbers, be an injection. If $f$ is a term involving all of the variables $x_{\varphi(0)}, \ldots, x_{\varphi(n-1)}$ and no others, then either $\mathscr{V}$ satisfies the identity

$$
f=0
$$

or there is an $\alpha \in E q v \mathbb{N}_{n}$ such that $\mathscr{V}$ satisfies the identity

$$
f=g\left(x_{\varphi(0)}, \ldots, x_{\varphi(n-1)}\right),
$$


where $g=\Phi_{n}(\alpha)$.

The details are left to the reader. We remark only that in both $\mathscr{B}$ and $\mathscr{B}^{\prime}$ we have the identities

$$
f+g=f g=0
$$

whenever the terms $f$ and $g$ have a variable in common. Thus, in view of identities (5) and (6), if any two subterms of $f$ have a variable in common, we get the identity

$$
f=0 \text {. }
$$

Now let $\mathscr{W}$ be a proper subvariety of $\mathscr{V}=\mathscr{B}$ or $\mathscr{B}^{\prime}$, and let

$$
f=g
$$

be an identity satisfied in $\mathscr{W}$ but not in $\mathscr{V}$. Without loss of generality, we may assume that $f$ is not constant in $\mathscr{V}$ and so that $f=f\left(x_{0}, \ldots, x_{n-1}\right)$ for $n \geq 1$, where all the variables $x_{0}, \ldots, x_{n-1}$ occur in $f$. Applying Lemma 4 to $f$, we get an $\alpha \in$ Eqv $\mathbb{N}_{n}$ such that $\mathscr{W}$ satisfies the identity

$$
\Phi_{n}(\alpha)\left(x_{0}, \ldots, x_{n-1}\right)=g,
$$

not satisfied by $\mathscr{V}$. If there is a variable occurring on one side of this identity and not the other, then, substituting 0 for that variable and applying Lemma 3, we see that $\Phi_{n}(\alpha)$ is constant in $\mathscr{W}$, that is, that $\mathscr{W}$ is not Bell. Otherwise, the variables $x_{0}, \ldots, x_{n-1}$ are precisely the variables occurring in $g$, and, applying Lemma 4 to $g$, we have a $\beta \in$ Eqv $\mathbb{N}_{n}$ such that the identity

$$
\Phi_{n}(\alpha)=\Phi_{n}(\beta)
$$

holds in $\mathscr{W}$ but not in $\mathscr{V}$. Then $\alpha \neq \beta$, and so $\Phi_{n}$ is not injective in $\mathscr{W}$, that is, again, $\mathscr{W}$ is not Bell. Thus no proper subvariety of $\mathscr{B}$ or $\mathscr{B}^{\prime}$ is Bell. In view of Lemma 1 , the proof of the theorem will be complete if we exhibit algebras in $\mathscr{B}$ and $\mathscr{B}^{\prime}$ in which, for each $n \geq 1$, the term operations in $\Phi_{n}\left(\right.$ Eqv $\left.\mathbb{N}_{n}\right)$ are all essentially $n$-ary and are all distinct. We proceed to this task.

Let the sets $F=F^{\prime}$ denote the set of all ordered pairs

$$
\langle\alpha, X\rangle
$$

where $X$ is a finite subset of $\mathbb{N}$ and $\alpha \in \operatorname{Eqv} X$. We define two algebraic structures $\mathbf{F}=\langle F,+, \cdot\rangle$ and $\mathbf{F}^{\prime}=\left\langle F^{\prime},+, \cdot\right\rangle$. In both $\mathbf{F}$ and $\mathbf{F}^{\prime}$ we set

$$
\langle\emptyset, \emptyset\rangle+\langle\alpha, X\rangle=\langle\alpha, X\rangle+\langle\emptyset, \emptyset\rangle=\langle\emptyset, \emptyset\rangle
$$


and

$$
\langle\emptyset, \emptyset\rangle\langle\alpha, X\rangle=\langle\alpha, X\rangle\langle\emptyset, \emptyset\rangle=\langle\emptyset, \emptyset\rangle
$$

for all $\langle\alpha, X\rangle$.

For any other pair $\langle\alpha, X\rangle,\langle\beta, Y\rangle$, that is, when $X \neq \emptyset, Y \neq \emptyset$, the sum is defined the same way in both algebras:

$$
\langle\alpha, X\rangle+\langle\beta, Y\rangle= \begin{cases}\langle\alpha \cup \beta, X \cup Y\rangle & \text { if } X \cap Y=\emptyset \\ \langle\emptyset, \emptyset\rangle & \text { if } X \cap Y \neq \emptyset\end{cases}
$$

where $\alpha \cup \beta$ is the ordinary set union, and has the same effect whether we regard $\alpha$ and $\beta$ as equivalence relations, that is, subsets of $X^{2}, Y^{2}$, respectively, or as partitions, that is, sets of subsets of $X, Y$, respectively; since the sets $X$ and $Y$ are disjoint, $\alpha \cup \beta \in \operatorname{Eqv}(X \cup Y)$.

The product is defined differently for $\mathbf{F}$ and $\mathbf{F}^{\prime}$. If $X \neq \emptyset, Y \neq \emptyset$, then in $\mathbf{F}$ we set

$(20)\langle\alpha, X\rangle\langle\beta, Y\rangle= \begin{cases}\left\langle\iota_{X \cup Y}, X \cup Y\right. & \text { if } X \cap Y=\emptyset \text { and } \alpha=\iota_{X}, \beta=\iota_{Y}, \\ \langle\emptyset, \emptyset\rangle & \text { otherwise, }\end{cases}$

where, for each set $X, \iota_{X}$ (or simply $\iota$ when the context is clear) denotes the equivalence relation $X^{2}$, equivalently, the partition with only one block.

For $\mathbf{F}^{\prime}$ we set

$$
\langle\alpha, X\rangle\langle\beta, Y\rangle= \begin{cases}\left\langle\iota_{X \cup Y}, X \cup Y\right\rangle & \text { if } X \cap Y=\emptyset \\ \langle\emptyset, \emptyset\rangle & \text { if } X \cap Y \neq \emptyset\end{cases}
$$

whenever $X \neq \emptyset$ and $Y \neq \emptyset$. Note that in both $\mathbf{F}$ and $\mathbf{F}^{\prime}$ we have

$$
\langle\emptyset, \emptyset\rangle=0
$$

\section{LEMMA 5. F $\in \mathscr{B}$ and $\mathbf{F}^{\prime} \in \mathscr{B}^{\prime}$}

ProOF. We need only show that F satisfies the identities (1)-(11) and that $\mathbf{F}^{\prime}$ satisfies the identities (1)-(10) and $\left(11^{\prime}\right)$. This is safely left to the reader. However, in order to preserve a modicum of honesty and at the dire risk of boring the reader, we present the verification of (4) for both $\mathbf{F}$ and $\mathbf{F}^{\prime}$, along with the verification of (11) for $\mathbf{F}$ and $\left(11^{\prime}\right)$ for $\mathbf{F}^{\prime}$.

We first verify (4). In both $\mathbf{F}$ and $\mathbf{F}^{\prime}$ both sides are $0=\langle\emptyset, \emptyset\rangle$ if at least one of $x, y$, or $z$ is 0 . Otherwise, we may set $x=\langle\alpha, X\rangle, y=\langle\beta, Y\rangle, z=\langle\gamma, Z\rangle$ 
for nonempty $X, Y, Z$. Then, for both $\mathbf{F}$ and $\mathbf{F}^{\prime}$ the left hand side of (4) is 0 unless $X \cap Y \neq \emptyset$ and $(X \cup Y) \cap Z \neq \emptyset$, that is, unless

$$
X \cap Y \neq \emptyset, X \cap Z \neq \emptyset, \text { and } Y \cap Z \neq \emptyset .
$$

From (20) it follows that in $\mathbf{F}$

$$
(x y) z= \begin{cases}\left\langle\iota_{X \cup Y \cup Z}, X \cup Y \cup Z\right\rangle & \text { if } X \cap Y=X \cap Z=Y \cap Z=\emptyset \\ 0 & \text { and } \alpha=\iota_{X}, \beta=\iota_{Y}, \gamma=\iota_{Z}, \\ & \text { otherwise, }\end{cases}
$$

which is exactly the computation for $x(y z)$.

Similarly, using (21), we compute in $\mathbf{F}^{\prime}$ that

$$
(x y) z= \begin{cases}\left\langle\iota_{X \cup Y \cup Z}, X \cup Y \cup Z\right\rangle & \text { if } X \cap Y=X \cap Z=Y \cap Z=\emptyset \\ 0 & \text { otherwise, }\end{cases}
$$

which is exactly the computation for $x(y z)$.

This verifies (4) in $\mathbf{F}$ and in $\mathbf{F}^{\prime}$.

We now verify (11) for $\mathbf{F}$. Again, we set $x=\langle\alpha, X\rangle, y=\langle\beta, Y\rangle, z=\langle\gamma, Z\rangle$. If one of $X, Y$ is $\emptyset$, then (11) follows immediately from (17) and (18). Otherwise, since $\alpha \cup \beta$ can then not be $\iota_{X \cup Y}$, we again get

$$
(x+y) z=0,
$$

concluding the verification of (11) for $F$.

To verify $\left(11^{\prime}\right)$ for $\mathbf{F}^{\prime}$ we note both sides are $\langle\emptyset, \emptyset\rangle$ unless $X, Y, Z$ are all nonempty and $X \cap Y=X \cap Z=Y \cap Z=\emptyset$, in which case

$$
x+y=\langle\alpha \cup \beta, X \cup Y\rangle
$$

and so

$$
(x+y) z=\left\langle\iota_{X \cup Y \cup Z}, X \cup Y \cup Z\right\rangle,
$$

which is precisely $x y z$, as derived above.

This concludes Lemma 5.

Now let $X$ be an $n$-element subset of $\mathbb{N}$ and let $\varphi: \mathbb{N}_{n} \rightarrow X$ be a bijection. For each $\alpha \in$ Eqv $X$ we denote by $\varphi^{*} \alpha$ the corresponding partition on $\mathbb{N}_{n}$,

$$
\varphi^{*} \alpha=\{\langle x, y\rangle \mid\langle\varphi(x), \varphi(y)\rangle \in \alpha\} .
$$

The following lemma is then immediate from the definitions: 
LEMMA 6. Let $X$ be an $n$-element subset of $\mathbb{N}$, let $\varphi: \mathbb{N}_{n} \rightarrow X$ be a bijection, and let $\alpha \in E q v X$. Then, in both the algebras $\mathbf{F}$ and $\mathbf{F}^{\prime}$,

$$
\langle\alpha, X\rangle=\Phi_{n}\left(\varphi^{*} \alpha\right)(\langle\iota,\{\varphi(0)\}\rangle, \ldots,\langle\iota,\{\varphi(n-1)\}\rangle) .
$$

We can now conclude the proof of the theorem. Indeed, it follows easily from Lemma 6 that $\mathbf{F}$ and $\mathbf{F}^{\prime}$ are Bell bisemigroups. Let $n \geq 1$ and let $\alpha \neq \beta$ be partitions of $\mathbb{N}_{n}$. Then, applying Lemma 6 with $\varphi$ the identity mapping, we get

$$
\begin{aligned}
\Phi_{n}(\alpha)(\langle\iota,\{0\}\rangle, \ldots,\langle\iota,\{n-1\}\rangle) & =\left\langle\alpha, \mathbb{N}_{n}\right\rangle \\
& \neq\left\langle\beta, \mathbb{N}_{n}\right\rangle \\
& =\Phi_{n}(\beta)(\langle\iota,\{0\}\rangle, \ldots,\langle\iota,\{n-1\}\rangle),
\end{aligned}
$$

that is, $\Phi_{n}$ is injective. The variables that occur in $\Phi_{n}(\alpha)$ are precisely $x_{0}, \ldots, x_{n-1}$. If $\Phi_{n}(\alpha)$ did not depend on one of these variables, then, substituting $0=\langle\emptyset, \emptyset\rangle$ for that variable and appealing to Lemma 3, we would derive the contradiction

$$
\langle\emptyset, \emptyset\rangle=\left\langle\alpha, \mathbb{N}_{n}\right\rangle .
$$

Thus the image of $\Phi_{n}$ is a subset of the essentially $n$-ary term functions of $F$, respectively of $\mathbf{F}^{\prime}$. That the image is precisely the set of essentially $n$-ary term functions follows immediately from Lemma 4 . Consequently, $\mathbf{F}$ and $\mathbf{F}^{\prime}$ are Bell bisemigroups, concluding the proof of the theorem.

For the proof of the Theorem it would suffice to state Lemma 6 with $X=$ $\mathbb{N}_{n}$ and $\varphi$ the identity map. We chose the more general statement because it then follows immediately that $\mathbf{F}$ and $\mathbf{F}^{\prime}$ are the free algebras generated by the countable set

$$
\{\langle\iota,\{n\}\rangle \mid n \in \mathbb{N}\}
$$

in $\mathscr{B}$ and $\mathscr{B}^{\prime}$, respectively.

\section{Concluding remarks}

In terms of the concept of $p_{n}$ sequence, [2], we have presented a representation of the sequence with

$$
p_{n}=B(n), \quad n \neq 1,
$$

and

$$
p_{1}=B(1)-1 \text {. }
$$


(The condition on $p_{1}$ is quite natural, since in any nontrivial algebra the term $x_{0}$ is always essentially unary $-p_{1}$ was defined, somewhat artificially, as 1 less than the number of essentially unary terms in order that, a priori, every sequence of natural numbers be possible.) What is new here is the naturality of the representation; since $p_{0} \neq 0$, the general theory of $p_{n}$ sequences trivially yields (nonnatural) representations of this sequence. Essentially, one introduces as many fundamental operations as one needs and then cuts down the number of essentially $n$-ary terms by identifying any extra terms to a constant. The variety $\mathscr{B}$ is very much in this spirit, but $\mathscr{B}^{\prime}$ is not.

A more interesting problem, and indeed not yet solved in general, is that of representing sequences with $p_{0}=p_{1}=0$, that is, characterizing the $p_{n}$ sequences of idempotent algebras with no constants. In the spirit of this paper, since $B(1)=1$, we can consider the problem of presenting the sequence

$$
\langle 0,0,2, \ldots, B(n), \ldots\rangle,
$$

that is, modifying our results so that we have no constants. However, general results in the literature show that this is impossible. Indeed, let us assume that the algebra $\mathbf{A}$ represents the above sequence. Since $B(2)=2$, $\mathbf{A}$ has exactly two essentially binary term functions. If one of them is commutative, then so is the other, that is, A has two commutative binary term operations. But then, by a result of Dudek [1],

$$
p_{3}(\mathbf{A}) \geq 9>5=B(3) .
$$

Thus, $\mathbf{A}$ has no binary commutative term functions. Then, by Kisielewicz [3, Theorem 4.1], there are natural numbers $a_{1}, a_{2}$ with

$$
p_{4}(\mathbf{A})=a_{1}\left(\begin{array}{l}
4 \\
1
\end{array}\right)+a_{2}\left(\begin{array}{l}
4 \\
3
\end{array}\right)
$$

consequently, $p_{4}$ is divisible by 4 , and so cannot be $B(4)=15$. Thus the sequence is not representable.

\section{References}

[1] J. Dudek, 'A characterization of distributive lattices', in: Contributions to Lattice Theory (Szeged 1980), volume 33 of Colloq. Math. Soc. János Bolyai (North-Holland, Amsterdam, 1983) pp. 325-335. 
[2] G. Grätzer, 'Composition of functions', in: Proceedings of the Conference on Universal Algebra, October 1969 (ed. G. H. Wenzel), Queen's Papers in Pure and Applied Mathematics (Queen's University, Kingston, 1970) pp. 1-106.

[3] A. Kisielewicz, 'Characterization of $p_{n}$-sequences for nonidempotent algebras', J. of Algebra 108 (1987), 102-115.

[4] R. N. McKenzie, G. F. McNulty and W. F. Taylor, in: Algebras, Lattices, Varieties Volume I, The Wadsworth \& Brooks/Cole Mathematics Series (Wadsworth \& Brooks/Cole, Monterey, 1987).

[5] R. P. Stanley, in: Enumerative Combinatorics Volume I, The Wadsworth \& Brooks/Cole Mathematics Series (Wadsworth \& Brooks/Cole, Monterey, 1986).

Department of Mathematics and Astronomy

University of Manitoba

Winnipeg, Canada R3T 2N2 